\title{
On a Division Algebra Classification of Constrained Generalized Supersymmetries
}

\section{Francesco Toppan*}

CBPF, Rio de Janeiro, Brazil

E-mail: toppan@cbpf.br

In this talk we present a division-algebra classification of the generalized supersymmetries admitting bosonic tensorial central charges. We show that for complex and quaternionic supersymmetries a whole class of compatible division-algebra constraints can be imposed. Possible applications to $M$-theory related dynamical systems are briefly mentioned.

Fourth International Winter Conference on Mathematical Methods in Physics 09 - 13 August 2004

Centro Brasileiro de Pesquisas Fisicas (CBPF/MCT), Rio de Janeiro, Brazil

\footnotetext{
* Speaker.
} 


\section{Introduction}

In this talk we present a work in progress, based on several papers of the author and his coworkers, concerning the division algebra classification of the generalized supersymmetries and of their consistent constraints.

This is an extremely important issue. We recall in fact that in the seventies the HŁS scheme [1] was a cornerstone providing the supersymmetric extension of the Coleman-Mandula no-go theorem. However, in the eighties [2] and especially in the nineties, the generalized space-time supersymmetries admitting bosonic tensorial central charges and going beyond the HŁS scheme found recognition [3,4] in association with the dynamics of extended objects like branes (see $[5,6])$. The eleven-dimensional $M$-algebra underlying the $M$-theory as a possible "Theory Of Everything" (TOE), admitting 32-real component spinors and maximal number $(=528)$ of saturated bosonic generators [3,4] falls into this class of generalized supersymmetries. This is the reason why a lot of attention has been recently devoted to the problem of classifying generalized supersymmetries, see e.g. [7] and [8]. A step towards this classification was provided in [9]. Based on the available classification of Clifford algebras and spinors in terms of division algebras [10, 11, 12], it was there shown that, in the complex and quaternionic cases, a division-algebra compatible constraint, leading to the two big classes of hermitian and holomorphic generalized supersymmetries, could be consistently imposed. In this talk we review the main ingredients entering the mathematical classification of generalized supersymmetries and present new results [13] on the classification of the constrained generalized supersymmetries.

The present paper is so conceived. In order to make it self-consistent, at first the divisionalgebra classification of Clifford algebras and fundamental spinors is recalled. The notion of "maximal Clifford algebras", essential for later developments, is introduced. It is explained how to recover all real, complex and quaternionic realizations in any given space-time from the set of fundamental maximal Clifford algebras which can be iteratively constructed. In the following, the notion of generalized supersymmetry is introduced in association with their division algebra properties. It is further explained how to implement various division algebra-compatible constraints, as well as their combinations. This amounts to introduce hermitian versus holomorphic constraints in the complex and quaternionic cases, as well as reality conditions implemented on bosonic generators. Some concrete examples of these division-algebra compatible constrained generalized supersymmetries are explicitly constructed. A series of tables with the main ingredients of the classification are presented. Finally, in the Conclusions, we will briefly mention the possible physical applications to supersymmetric dynamical systems (and their relation with the $M$-theory) of the above construction.

\section{Basic notions: division algebras, Clifford algebras and fundamental spinors}

The four division algebras of real $(\mathbf{R})$ and complex $(\mathbf{C})$ numbers, quaternions $(\mathbf{H})$ and octonions $(\mathbf{O})$ possess respectively $0,1,3$ and 7 imaginary elements $e_{i}$ satisfying the relations

$$
e_{i} \cdot e_{j}=-\delta_{i j}+C_{i j k} e_{k}
$$

$(i, j, k$ are restricted to take the value 1 in the complex case, 1,2,3 in the quaternionic case and $1,2, \ldots, 7$ in the octonionic case; furthermore, the sum over repeated indices is understood). 
$C_{i j k}$ are the totally antisymmetric division-algebra structure constants. The octonionic division algebra is the maximal, since quaternions, complex and real numbers can be obtained as its restriction. The totally antisymmetric octonionic structure constants can be expressed as

$$
C_{123}=C_{147}=C_{165}=C_{246}=C_{257}=C_{354}=C_{367}=1
$$

(and vanishing otherwise).

The octonions are the only non-associative, however alternative (see [14]), division algebra.

For our later purposes it is of particular importance the notion of division-algebra principal conjugation. Any element $X$ in the given division algebra can be expressed through the sum

$$
X=x_{0}+x_{i} e_{i}
$$

where $x_{0}$ and $x_{i}$ are real, the summation over repeated indices is understood and the positive integral $i$ are restricted up to 1, 3 and 7 in the $\mathbf{C}, \mathbf{H}$ and $\mathbf{O}$ cases respectively. The principal conjugate $X^{*}$ of $X$ is defined to be

$$
X^{*}=x_{0}-x_{i} e_{i}
$$

It allows introducing the division-algebra norm through the product $X^{*} X$. The normed-one restrictions $X^{*} X=1$ select the three parallelizable spheres $S^{1}, S^{3}$ and $S^{7}$ in association with $\mathbf{C}, \mathbf{H}$ and $\mathbf{O}$ respectively.

For what concerns the main properties of Clifford algebras and their relation with the associative division algebras $\mathbf{R}, \mathbf{C}, \mathbf{H}$ it is convenient to follow [12] and [15].

The most general irreducible real matrix representations of the Clifford algebra

$$
\Gamma^{\mu} \Gamma^{v}+\Gamma^{v} \Gamma^{\mu}=2 \eta^{\mu \nu}
$$

with $\eta^{\mu \nu}$ being a diagonal matrix of $(p, q)$ signature (i.e. $p$ positive, +1 , and $q$ negative, -1 , diagonal entries) ${ }^{1}$ can be classified according to the property of the most general $S$ matrix commuting with all the $\Gamma^{\prime}$ 's $\left(\left[S, \Gamma^{\mu}\right]=0\right.$ for all $\left.\mu\right)$. If the most general $S$ is a multiple of the identity, we get the normal $(\mathbf{R})$ case. Otherwise, $S$ can be the sum of two matrices, the second one multiple of the square root of -1 (this is the almost complex, $\mathbf{C}$ case) or the linear combination of 4 matrices closing the quaternionic algebra (this is the $\mathbf{H}$ case).

For our purposes the division-algebra character of the Clifford irreps can be understood as follows. In the $\mathbf{R}$-case the matrices realizing the irrep have necessarily real entries, in the $\mathbf{C}$-case matrices with complex entries can be used, while in the $\mathbf{H}$-case the matrices can be realized with quaternionic entries.

Let us see how this works in a simple example. Let us take the $\mathbf{H}$-type $C(0,3)$ Clifford algebra. It can be realized by associating the three Euclidean gamma matrices with the three imaginary quaternions $e_{i}$. The reason for that lies on the fact that the antisimmetry of the $C_{i j k}(2.1)$ structure constants make the anticommutators $e_{i} e_{j}+e_{j} e_{i}$ satisfy the relation

$$
e_{i} e_{j}+e_{j} e_{i}=-\delta_{i j}
$$

\footnotetext{
${ }^{1}$ Throughout this paper it will be understood that the positive eigenvalues are associated with space-like directions, the negative ones with time-like directions.
} 
reproducing the three dimensional Euclidean Clifford algebra (2.5) with negative signs.

It is worth mentioning that in the given signatures $p-q \bmod 8=0,4,6,7$, without loss of generality, the $\Gamma^{\mu}$ matrices can be chosen block-antidiagonal (generalized Weyl-type matrices), i.e. of the form

$$
\Gamma^{\mu}=\left(\begin{array}{cc}
0 & \sigma^{\mu} \\
\tilde{\sigma}^{\mu} & 0
\end{array}\right)
$$

Since the generalized Lorentz algebra can be recovered from the algebra of the commutators $\Sigma^{[\mu v]}=\left[\Gamma^{\mu}, \Gamma^{v}\right]$, in those particular signatures the matrices $\Sigma^{[\mu v]}$ are of block-diagonal type and it is therefore possible to introduce Weyl-projected spinors, whose number of components is half of the size of the corresponding $\Gamma$-matrices (this notion of Weyl spinors, which is convenient for our purposes, has been introduced in [15]).

In [15] the representatives of all Clifford irreps in any given space-time were systematically constructed with the help of two recursive algorithms (producing $D+2$-dimensional Clifford irreps from $D$-dimensional spacetime Clifford irreps), to be applied to the solutions (such as (2.6)) of the equation (2.5) in terms of imaginary elements of a division algebra (see [15] for detail).

Let us briefly comment about the octonionic realization of the (2.5) relation, through matrices admitting octonionic entries. Since the octonions are non-associative, this realization presents peculiar features. In [16] and [17] it was shown how it could be associated with an octonionic version of the $M$ algebra and its associated superconformal algebra. Throughout this paper we will limit ourselves to consider only standard, associative, Clifford algebras representations.

Fundamental spinors carry a representation of the generalized Lorentz group with a minimal number of real components in association with the maximal, compatible, allowed division-algebra structure (they can be thought as column vectors with entries in the given division algebra).

It is worth reminding that the division-algebra character of fundamental spinors does not necessarily (depending on the given space-time) coincide with the division-algebra type of the corresponding Clifford irreps (this mismatch lies on the fact that in some given spacetimes the fundamental spinors are of Weyl type). In different space-times parametrized by $\rho=s-t \bmod 8$, fundamental spinors can accommodate for $\rho=2,3$ a larger division-algebra structure than the corresponding Clifford irreps. Conversely, for $\rho=6,7$, the Clifford irreps accommodate a larger division-algebra structure than the corresponding spinors.

\section{Maximal Clifford algebras and their reductions}

An extremely useful notion is that of "maximal Clifford algebra" (see [15]). "Maximal Clifford algebras" correspond to the Clifford irreps which can accommodate the maximal number of Gamma matrices for the corresponding size of the matrices. Stated otherwise,for any given spacetime, its Clifford irrep can be obtained from its associated maximal Clifford algebras. Non-maximal Clifford algebras are simply recovered after deleting a certain number of Gamma matrices from a given maximal one (a procedure which parallels the dimensional reduction).

The knowledge of maximal Clifford algebras (which can be obtained with the lifting algorithms of [15]) allows us to reconstruct the full set of Clifford irreps in any given space-time.

$004 / 4$ 
The maximal Clifford irreps exist iff $(p-q)=1,5 \bmod \quad 8$. The $(p-q)=1 \bmod 8$ condition corresponds to a real case, while the $p-q=5 \bmod 8$ condition corresponds to a quaternionic case. The non-maximal Clifford algebras, given by $p-q \neq 1,5 \bmod 8$, can be recovered with the procedure illustrated by the following table [13], specifying real, complex and quaternionic Clifford irreps (denoted as $\Gamma$ ) and Clifford representations (not necessarily irrep, denoted as $\Psi$ ) supporting fundamental spinors:

\begin{tabular}{|lcc|l|l|}
\hline & & 1 & mod $8 \quad(\mathbf{R})$ & $5 \quad \bmod \quad 8 \quad(\mathbf{H})$ \\
\hline 0 & mod & 8 & $\Gamma, \Psi:(p, q) \stackrel{W}{\rightarrow}(p-1, q)$ & \\
\hline 4 & mod & 8 & & $\Gamma, \Psi:(p, q) \stackrel{W}{\rightarrow}(p-1, q)$ \\
\hline 2 & mod & 8 & $\Gamma:(p, q) \rightarrow(p, q-1)$ & $\Psi:(p, q) \stackrel{*}{\rightarrow}(p-2, q) \stackrel{W}{\rightarrow}(p-3, q)$ \\
\hline 3 & mod & 8 & & $\begin{array}{l}\Gamma:(p, q) \stackrel{*}{\rightarrow}(p-2, q) \\
\Psi:(p, q) \stackrel{W}{\rightarrow}(p-2, q)\end{array}$ \\
\hline 6 & & & & $\Gamma:(p, q) \rightarrow(p, q-1)$ \\
& & & & $\Psi:(p, q) \stackrel{*}{\rightarrow}(p, q-2) \stackrel{W}{\rightarrow}(p-1, q-2)$ \\
\hline 7 & mod & 8 & $\Psi:(p, q) \stackrel{W}{\rightarrow}(p-2, q)$ & $\Gamma:(p, q) \stackrel{*}{\rightarrow}(p, q-2)$ \\
\hline
\end{tabular}

Some remarks are in order. The real case is shown in the second column, while both the complex and the quaternionic cases are recovered from the third column. The arrows denote which gamma matrices (either space-like or time-like) and how many of them have to be deleted from the corresponding maximal Clifford algebra. The " $W$ " symbol above an arrow specifies whether the Weyl projection is required in order to produce fundamental spinors, while the " $*$ " symbol above an arrow denotes a reduction to the complex case.

The $(p, q) \stackrel{*}{\rightarrow}(p-2, q)$ reduction can only be performed under the condition $p \geq 3$, see [13] for details. Similarly, the $(p, q) \stackrel{*}{\rightarrow}(p, q-2)$ reduction requires $q \geq 3$ (all cases of physical interest enter the above table, the remaining few exceptional cases can be treated separately).

\section{On generalized supersymmetries}

Let us introduce now the notion of generalized supersymmetries as an extension and generalization of the standard supertranslation algebra (in some cases, like the $F$-algebra presentation in a $(10,2)$ spacetime of the $M$-algebra [9], the bosonic sector admits no translation at all, but still it is convenient to refer to generalized supersymmetries as "generalized supertranslations"). Generalized supertranslations can be used as building blocks to construct superconformal algebras (by simply taking two separate copies of generalized supertranslations and then imposing the closure of the super-Jacobi identities on all generators, [16]). Once obtained a generalized superconformal algebra, generalized superPoincaré algebras admitting, besides the generalized supertranslations, also the generalized Lorentz generators, can be recovered through an Inonü-Wigner contraction procedure. Throughout this paper we will focus just on the building blocks, namely the generalized supertranslations.

At first we need to introduce two matrices, denoted as $A$ and $C[18]$, related with, respectively, the hermitian conjugation and transposition acting on Gamma matrices. A plays the role of the time-like $\Gamma^{0}$ matrix in the Minkowskian space-time and is used to introduce barred spinors. $C$, on 
the other hand, is the charge conjugation matrix. Up to an overall sign, in a generic $(p, q)$ spacetime, $A$ and $C$ are given by the products of all the time-like and, respectively, all the symmetric (or antisymmetric) Gamma-matrices (depending on the given space-time there are at most two charge conjugations matrices, $C_{S}, C_{A}$, given by the product of all symmetric and all antisymmetric gamma matrices). For our purposes the importance of $A$ and the charge conjugation matrix $C$ lies on the fact that, in a $D$-dimensional space-time $(D=p+q)$ spanned by $d \times d$ Gamma matrices, they allow to construct a basis for $d \times d$ (anti)hermitian and (anti)symmetric matrices, respectively. The $\left(\begin{array}{l}D \\ k\end{array}\right)$ antisymmetrized products of $k$ Gamma matrices $A \Gamma^{\left[\mu_{1} \ldots \mu_{k}\right]}$ are all hermitian or all antihermitian, depending on the value of $k \leq D$. Similarly, the antisymmetrized products $C \Gamma^{\left[\mu_{1} \ldots \mu_{k}\right]}$ are all symmetric or all antisymmetric.

A generalized supersymmetry algebra involving $n$-component real spinors $Q_{a}$ is given by the anticommutators

$$
\left\{Q_{a}, Q_{b}\right\}=Z_{a b}
$$

where the matrix $Z$ appearing in the r.h.s. is the most general $n \times n$ symmetric matrix with total number of $\frac{n(n+1)}{2}$ components. For any given space-time we can easily compute its associated decomposition in terms of the antisymmetrized products of $k$-Gamma matrices, namely

$$
Z_{a b}=\sum_{k}\left(C \Gamma_{\left[\mu_{1} \ldots \mu_{k}\right]}\right)_{a b} Z^{\left[\mu_{1} \ldots \mu_{k}\right]}
$$

where the values $k$ entering the sum in the r.h.s. are restricted by the symmetry requirement for the $a \leftrightarrow b$ exchange and are specific for the given spacetime. The coefficients $Z^{\left[\mu_{1} \ldots \mu_{k}\right]}$ are the rank- $k$ abelian tensorial central charges.

In the case of Weyl projected spinors $\widetilde{Q}_{a}$ the r.h.s. has to be reconstructed with the help of a projection operator which selects the upper left block in a $2 \times 2$ block decomposition. Specifically, if $Z$ is a matrix decomposed in $2 \times 2$ blocks as $Z=\left(\begin{array}{cc}z_{1} & z_{2} \\ z_{3} & z_{4}\end{array}\right)$, we can define

$$
P(Z) \equiv Z_{1}
$$

The generalized supersymmetry algebra in the Weyl case can be expressed through

$$
\left\{\widetilde{Q}_{a}, \widetilde{Q}_{b}\right\}=P(Z)_{a b}
$$

A complex (quaternionic) generalized supersymmetry algebra is expressed in terms of complex (quaternionic) spinors $Q_{a}$ and their conjugate $Q^{*}{ }_{a}$. The most general (with a saturated r.h.s.) algebra is in this case given by

$$
\left\{Q_{a}, Q_{b}\right\}=\mathcal{P}_{a b} \quad, \quad\left\{Q^{*}{ }_{a}, Q_{\dot{b}}^{*}\right\}=\mathcal{P}^{*} \dot{a} \dot{b},
$$

together with

$$
\left\{Q_{a}, Q^{*}{ }_{b}\right\}=\mathcal{R}_{a b},
$$


where the matrix $\mathcal{P}_{a b}\left(\mathcal{P}^{*}{ }_{a} \dot{b}\right.$ is its conjugate and does not contain new degrees of freedom $)$ is symmetric, while $\mathcal{R}_{a b}$ is hermitian.

The maximal number of allowed components in the r.h.s. is given, for complex fundamental spinors with $n$ complex components, by $n(n+1)$ (real) bosonic components entering the symmetric $n \times n$ complex matrix $\mathcal{P}_{a b}$ plus $n^{2}$ (real) bosonic components entering the hermitian $n \times n$ complex matrix $\mathcal{R}_{a b}$.

A Weyl projection similar to (4.4) can be applied for complex and quaternionic spinors as well.

\section{Real generalized supersymmetries}

In this section we present a series of tables, taken from [9] and [13], listing the main properties of real generalized supersymmetries.

It is convenient to symbolically denoted as " $M_{k}$ " the space of $\left(\begin{array}{c}D \\ k\end{array}\right)$-component, totally antisymmetric rank- $k$ tensors of a $D$-dimensional spacetime, associated to the basis of the symmetric matrices $C \Gamma^{\left[\mu_{1} \ldots \mu_{k}\right]}$.

In the case of generalized real supersymmetries, depending on the dimensionality $D$ of the space-time (and independently from its signature, provided that the spinors admit the same minimal number of components), the bosonic sector, together with its number of bosonic components, is reported in the following table. Since maximal Clifford algebras are odd-dimensional, without loss of generality only odd dimensions $D$ enter the table below

\begin{tabular}{|c|c|c|}
\hline spacetime & bosonic sectors & bosonic components \\
\hline$D=1$ & $M_{0}$ & 1 \\
\hline$D=3$ & $M_{1}$ & 3 \\
\hline$D=5$ & $M_{2}$ & 10 \\
\hline$D=7$ & $M_{0}+M_{3}$ & $1+35=36$ \\
\hline$D=9$ & $M_{0}+M_{1}+M_{4}$ & $1+9+126=136$ \\
\hline$D=11$ & $M_{1}+M_{2}+M_{5}$ & $11+55+462=528$ \\
\hline$D=13$ & $M_{2}+M_{3}+M_{6}$ & $78+286+1716=2080$ \\
\hline
\end{tabular}

Generalized supersymmetries in even dimensional spacetime can be obtained from the previous list via a dimensional reduction (by erasing some Gamma matrices, as explained in Section 3). We obtain that the dimensional reduction $D \rightarrow D-1$ corresponding to the signature passage $(p, q) \rightarrow$ $(p, q-1)$ (here $D=p+q)$ is expressed through

\begin{tabular}{|l|l|l|}
\hline spacetime & bosonic sectors & bosonic components \\
\hline$D=3$ & $M_{1} \rightarrow \bar{M}_{1}+\bar{M}_{0}$ & $3=2+1$ \\
\hline$D=5$ & $M_{2} \rightarrow \bar{M}_{2}+\bar{M}_{1}$ & $10=6+4$ \\
\hline$D=7$ & $M_{0}+M_{3} \rightarrow \bar{M}_{0}+\bar{M}_{3}+\bar{M}_{2}$ & $36=1+20+15$ \\
\hline$D=9$ & $M_{0}+M_{1}+M_{4} \rightarrow 2 \times \bar{M}_{0}+\bar{M}_{1}+\bar{M}_{4}+\bar{M}_{3}$ & $136=2+8+70+56$ \\
\hline$D=11$ & $M_{1}+M_{2}+M_{5} \rightarrow \bar{M}_{0}+\bar{M}_{2} \times \bar{M}_{1}+\bar{M}_{2}+\bar{M}_{4}+\bar{M}_{5}$ & $528=1+20+45+210+252$ \\
\hline$D=13$ & $M_{2}+M_{3}+M_{6} \rightarrow \bar{M}_{1}+2 \times \bar{M}_{2}+\bar{M}_{3}+\bar{M}_{5}+\bar{M}_{6}$ & $2080=12+2 \times 66+220+792+924$ \\
\hline
\end{tabular}


The overlined quantities $\bar{M}_{k}$ are referred to the totally antisymmetric $k$-tensors in the $D-1$ dimensional spacetime.

It is also convenient to illustrate the dimensional reduction leading from the $(p, q) \rightarrow(p-1, q)$ spacetime. The difference w.r.t. the previous case lies on the fact that now the $(p-1, q)$ spacetime is of Weyl type (confront the discussion in Section 2). Only the subclass of totally antisymmetric bosonic $k$-tensors entering the upper left diagonal block will survive from the Weyl projection and enter the generalized supersymmetry. The corresponding symbols are marked in boldface $\left(\mathbf{M}_{k}\right)$ in the table below, corresponding to the even-dimensional Weyl case

\begin{tabular}{|l|l|l|}
\hline spacetime & bosonic sectors & bosonic components \\
\hline$D=2$ & $M_{0}+\frac{1}{2} \mathbf{M}_{1}$ & 1 \\
\hline$D=4$ & $\frac{1}{2} \mathbf{M}_{2}+M_{1}$ & 3 \\
\hline$D=6$ & $M_{0}+\frac{1}{2} \mathbf{M}_{3}+M_{2}$ & 10 \\
\hline$D=8$ & $\mathbf{M}_{0}+M_{1}+M_{3}+\frac{1}{2} \mathbf{M}_{4}$ & $36=1+35$ \\
\hline$D=10$ & $M_{0}+\mathbf{M}_{1}+M_{2}+M_{4}+\frac{1}{2} \mathbf{M}_{5}$ & $136=10+126$ \\
\hline$D=12$ & $M_{1}+\mathbf{M}_{2}+M_{3}+M_{5}+2+\frac{1}{2} \mathbf{M}_{6}$ & $528=66+462$ \\
\hline
\end{tabular}

In the above table the factor $\frac{1}{2}$ has been inserted to remind that $\mathbf{M}_{\frac{D}{2}}$ is self-dual, so that its total number of components has to be halved in order to fulfill the selfduality condition.

\section{Constrained complex generalized supersymmetries}

Two big classes of subalgebras, respecting the Lorentz-covariance, can be obtained from (4.5) and (4.6) in both the complex and quaternionic cases, by setting identically equal to zero either $\mathcal{P}$ or $\mathcal{R}$, namely assuming that either

$$
\mathcal{P}_{a b} \equiv \mathcal{P}^{*} \dot{a} \dot{b} \equiv 0,
$$

so that the only bosonic degrees of freedom enter the hermitian matrix $\mathcal{R}_{a b}$ or, conversely, that

$$
\mathcal{R}_{a b} \equiv 0,
$$

so that the only bosonic degrees of freedom enter $\mathcal{P}_{a b}$ and its conjugate matrix $\mathcal{P}^{*}{ }_{a} \dot{b}$.

Following [9] we will refer to the (complex or quaternionic) generalized supersymmetries satisfying the first constraint as "hermitian" generalized supersymmetries, while the (complex or quaternionic) generalized supersymmetries satisfying the second constraint will be referred to as "holomorphic" generalized supersymmetries. This distinction finds application in physics. It was proven in [19] that the analytical continuation of the $M$-algebra can be carried out to the Euclidean, the corresponding Euclidean algebra being a complex holomorphic supersymmetry.

Further refinements in the classification of division algebra constrained generalized supersymmetries can be produced by allowing a reality (or imaginary) constraint on the bosonic matrices $\mathcal{P}$ and $\mathcal{R}$. It is convenient to illustrate it by discussing, at first, some specific examples of interest, for later producing general results. 
Let us start describing the generalized supersymmetries associated with the $(4,1)$ space-time. Its fundamental spinors are quaternionic and admit 8 real components. We are in the position to classify all real and complex saturated generalized supersymmetries associated to this spacetime (the quaternionic supersymmetries are introduced in the next section). There are seven separated cases that we are able to consider, depending on the mixed conditions (holomorphicity, hermiticity, reality or imaginary constraint on $\mathcal{P}$ and $\mathcal{R}$ ) that can be imposed. The complete class of constrained generalized supersymmetries can be given as follows:

i) Real generalized supersymmetry with 36 bosonic components. This real generalized supersymmetry can also be expressed in the complex spinor formalism, the 36 bosonic components being recovered from $36=20+16$, the sum (in the real counting) of the holomorphic and hermitian sectors of the bosonic r.h.s.,

ii) A constrained complex supersymmetry obtained by imposing a reality condition on $\mathcal{R}$ alone. The total number of bosonic components in this case is 30 ,

iii) The constraint arising by impoing either a reality condition on $\mathcal{P}$ or, altenatively, an imaginary condition on $\mathcal{R}$. The total number of bosonic components is 26 ,

iv) The holomorphically constrained complex generalized supersymmetry with 20 bosonic components in the real counting (alternatively described by a reality condition on both $\mathcal{P}$ and $\mathcal{R}$,

v) The hermitian complex generalized supersymmetry with 16 bosonic components (real counting) (alternatively described by a reality condition on $\mathcal{P}$ and an imaginary condition on $R$ ),

$v i)$ The holomorphically (or hermitian) constrained complex generalized supersymmetry with reality condition on the bosonic r.h.s., leading to $\frac{1}{2} \times 20=10$ bosonic components and, finally,

vii) the hermitian supersymmetry with an imaginary constraint on the bosonic sector, leading to 6 bosonic components.

The generalized supersymmetries for $(4,1)$ allow us to immediately construct the generalized supersymmetries in the standard Minkowskian $(3,1)$ space-time, which can be obtained as a Weyl-type dimensional reduction from $(4,1)$, see table (3.1). The corresponding generalized supersymmetries in this case admit a total number of bosonic generators, whose counting, due to the Weyl condition based on 4-component spinors, is given by the following list

i) 10 in the real case $(10=6+4$, in the complex presentation),

ii) 9 in this real $\mathcal{R}$ case,

iii) 7 in this real $\mathcal{P}$ case,

iv) 6 in this case, corresponding to the complex hermitian supersymmetry,

v) 4 in this case, corresponding to the complex holomorphic supersymmetry,

vi) 3 for a hermitian or holomorphic supersymmetry supplemented by a reality condition,

vii) 1 for a holomorphic supersymmetry with imaginary $\mathcal{P}$.

The above classes of supersymmetries are present in all cases when the fermionic generators are realized through complex spinors.

Both the $(4,1)$ and the $(3,1)$ spacetimes are not maximal Clifford algebras. The maximal Clifford algebras associated to them are recovered from the (3.1) table. The above list of generalized supersymmetries finds immediate application in the construction of all possible constrained dynamical systems arising from dimensional reduction of one given system associated to the maximal Clifford spacetime (examples of such systems are the particle models admitting tensorial central charges, briefly discussed in the Conclusion). This explains the importance of both the (3.1) table 
(for the derivation of maximal Clifford algebras) and of the above constraints in the classification of generalized supersymmetries.

The above results can be extended to any kind of generalized supersymmetries admitting $n$ component complex spinors (i.e. $2 n$ distinct components in the real counting). In the following table the associated generalized supersymmetries are listed, as well as the total number of bosonic (real-counting) degrees of freedom. We have

\begin{tabular}{|l|l|l|}
\hline$i$ & Real supersymmetry & $2 n^{2}+n$ bosonic components \\
\hline$i i$ & reality on $\mathcal{R}$ & $\frac{3}{2}\left(n^{2}+n\right)$ bosonic components \\
\hline iii & reality on $P$ & $\frac{1}{2}\left(3 n^{2}+n\right)$ bosonic components \\
\hline$i v$ & complex holomorphic supersymmetry & $n^{2}+n$ bosonic components \\
\hline$v$ & complex hermitian & $n^{2}$ bosonic components \\
\hline$v i$ & complex holomorphic with bosonic reality constraint & $\frac{1}{2}\left(n^{2}+n\right)$ bosonic components \\
\hline$v i i$ & complex holomorphic with bosonic imaginary constraint & $\frac{1}{2}\left(n^{2}-n\right)$ bosonic components \\
\hline
\end{tabular}

The constrained generalized supersymmetries labeled by iii, $i v, v$ and $v i$ admit an equivalent, dual presentation. We discussed this feature in the concrete example of the Minkowskian $(4,1)$ generalized supersymmetries, but this is a general property, valid in any space-time signature. The dual relations are given by

iii) $\mathcal{P}$ real $\leftrightarrow \mathcal{R}$ imaginary,

iv) $\mathcal{R}=0 \leftrightarrow \mathcal{P}, \mathcal{R}$ bothreal,

v) $\mathcal{P}=0 \leftrightarrow \mathcal{P}$ real, $\mathcal{R}$ imaginary,

vi) Preal, $\mathcal{R}=0 \leftrightarrow R$ real, $\mathcal{P}=0$.

\section{Generalized supersymmetries of the quaternionic spacetimes}

For what concerns the complex and quaternionic cases several tables can be produced presenting the complete list of the associated constrained supersymmetries. For lack of space we limit ourselves to reproduce here some selected examples concerning the generalized supersymmetries supported by quaternionic spacetimes. This provides a reader with an idea about the main features of the classification.

The first case we present corresponds to the hermitian quaternionic supersymmetry, whose fermionic generators are quaternionic spinors (the corresponding spacetimes supporting such spinors and their associated supersymmetries are given in ([15])). In this particular case the corresponding 
table is given by

\begin{tabular}{|c|c|c|}
\hline spacetime & bosonic sectors & bosonic components \\
\hline$D=3$ & $M_{0}$ & 1 \\
\hline$D=4$ & $M_{0}$ & 1 \\
\hline$D=5$ & $M_{0}+M_{1}$ & $1+5=6$ \\
\hline$D=6$ & $M_{1}$ & 6 \\
\hline$D=7$ & $M_{1}+M_{2}$ & $7+21=28$ \\
\hline$D=8$ & $M_{2}$ & 28 \\
\hline$D=9$ & $M_{2}+M_{3}$ & $36+84=120$ \\
\hline$D=10$ & $M_{3}$ & 120 \\
\hline$D=11$ & $M_{0}+M_{3}+M_{4}$ & $1+165+330=496$ \\
\hline$D=12$ & $M_{0}+M_{4}$ & $1+495=496$ \\
\hline$D=13$ & $M_{0}+M_{1}+M_{4}+M_{5}$ & $1+13+715+1287=2016$ \\
\hline
\end{tabular}

As an example of holomorphic supersymmetry we produce a table corresponding to the complex holomorphic supersymmetry for quaternionic spacetime, i.e. carrying a quaternionic structure, however expressing spinors only through their complex structure. This implies that the reality condition on the bosonic sector is automatically implemented. Some similarities should be observed between the table (5.1) and the table below. They correspond however to different cases, real versus complex holomorphic supersymmetries, associated to spacetimes with different signatures and different number of spinorial components (in the complex holomorphic case the number of spinor components are double than in the real case, for $D$-dimensional spacetimes). Their similarities on the other hand have a very deep physical meaning. They imply, e.g., that the complex holomorphic supersymmetry can be used to perform the analytic continuation of real supersymmetries to different signatures (the Euclideanized version of the $M$-algebra, see [19], corresponds to the analytical continuation of the real $M$ algebra). We have now

\begin{tabular}{|c|c|c|}
\hline spacetime & bosonic sectors & bosonic components \\
\hline$D=3$ & $M_{1}$ & 3 \\
\hline$D=4$ & $\widetilde{M}_{2}$ & 3 \\
\hline$D=5$ & $M_{2}$ & 10 \\
\hline$D=6$ & $\widetilde{M}_{3}$ & 10 \\
\hline$D=7$ & $M_{0}+M_{3}$ & $1+35=36$ \\
\hline$D=8$ & $M_{0}+\widetilde{M}_{4}$ & $1+35=36$ \\
\hline$D=9$ & $M_{0}+M_{1}+M_{4}$ & $1+9+126=136$ \\
\hline$D=10$ & $M_{1}+\widetilde{M}_{5}$ & $10+126=136$ \\
\hline$D=11$ & $M_{1}+M_{2}+M_{5}$ & $11+55+462=528$ \\
\hline$D=12$ & $M_{2}+\widetilde{M}_{6}$ & $66+462=528$ \\
\hline$D=13$ & $M_{2}+M_{3}+M_{6}$ & $78+286+1716=2080$ \\
\hline
\end{tabular}

The classification of the (full) quaternionic holomorphic supersymmetry, which presents peculiar features, has been given and discussed in [9]. The results can be summarized as follows 


\begin{tabular}{|c|lcc|}
\hline- & $D=0,6,7$ & $\bmod$ & 8 \\
\hline$M_{0}$ & $D=1$ & $\bmod$ & 8 \\
\hline$M_{1}$ & $D=4,5$ & $\bmod$ & 8 \\
\hline$M_{0}+M_{1}$ & $D=2,3$ & $\bmod$ & 8 \\
\hline
\end{tabular}

The above results can be interpreted as follows. Quaternionic holomorphic supersymmetries only arise in $D$-dimensional quaternionic space-times, where $D=2,3,4,5 \bmod \quad 8$. No such supersymmetry exists in $D=0,6,7$ mod $8 D$-dimensional spacetimes.

In $D=1 \bmod 8$ dimensions it only involves a single bosonic charge and falls into the class of quaternionic supersymmetric quantum mechanics, rather than supersymmetric relativistic theories. Finally, this supersymmetry algebra only admits at most a scalar bosonic central charge, found in $D$-dimensional quaternionic spacetimes for $D=2,3 \bmod 8$.

It must be said that so far no dynamical system supporting such a supersymmetry has been investigated.

\section{Conclusions}

This paper was devoted to perform a division algebra classification of the generalized supersymmetries. Besides the notion of hermitian (complex and quaternionic) and holomorphic (complex and quaternionic) supersymmetries, already presented in [9], a further distinction of divisionalgebra constrained generalized supersymmetries, given by table (6.3), has been presented. This set of constrained supersymmetries corresponds to certain classes of division algebra constraints that can be consistently imposed (e.g., a reality condition on the bosonic sector of complex holomorphic supersymmetries ). The sets of constraints can even be combined together, as discussed in Section 6.

Another issue that we have here clarified consists in the explicit construction, see table (3.1), of the non-maximal Clifford algebras and their associated spinors, in terms of their associated maximal Clifford algebras. The two main new results here presented allow to classify and put in a single framework (via dimensional reduction), showing their web of inter-related dualities, a whole class of generalized supersymmetries. They can be combined to produce, on a physical side, the largest "oxydized" dynamical system which can be regarded as the generator of all reduced and constrained lowest dimensional models.

Some of the mathematical issues here discussed have already been employed to, e.g., performing the analytic continuation of the $M$ algebra [19] (it corresponds to an eleven-dimensional complex holomorphic supersymmetry and in [9] it was further shown that the same algebra also admits a 12-dimensional Euclidean presentation in terms of Weyl-projected spinors). These two examples of Euclidean supersymmetries can find application in the functional integral formulation of higher-dimensional supersymmetric models.

There is an interesting class of models which nicely fits in the framework here described and is currently under intense investigation. It is the class of superparticle models, introduced at first in [20] and later studied in [21], whose bosonic coordinates correspond to tensorial central charges.

$004 / 12$ 
It was shown in [22] that a 4-dimensional theory of this kind leads to a tower of massless higher spin states, concretely implementing a Fronsdal's proposal [23] of introducing bosonic tensorial coordinates to describe massless higher spin theories (admitting helicity states greater than two). This is an active area of research, the main motivation being the investigation of the tensionless limit of superstring theory, corresponding to a tower of higher helicity massless particles (see e.g. [24]).

In a somehow "orthogonal" direction, a class of theories which can be investigated in the present framework is the class of supersymmetric extensions of Chern-Simon supergravities in higher dimensions, requiring as a basic ingredient a Lie superalgebra admitting a Casimir of appropriate order, see e.g. [25].

\section{References}

[1] R. Haag, J. Łopuszański and M. Sohnius, All Possible Generators of Supersymmetries of the S Matrix, Nucl. Phys. B88 (1975) 257.

[2] R. D'Auria and P. Fré, Geometric Supergravity in D=11 and its Hidden Supergroup, Nucl. Phys. B201 (1982) 101, erratum ibid. B206 (1982) 496.

[3] J. A. de Azcarraga, J. P. Gauntlett, J. M. Izquierdo and P. K. Townsend, Topological Extensions of the Supersymmetry Algebra for Extended Objects, Phys. Rev. Lett. 63 (1989) 2443.

[4] P. Townsend, M Theory from its Superalgebra, Cargese Lectures, 1997 [hep-th/9712004].

[5] R. Güven, Black p-Brane Solutions of D = 11 Supergravity, Phys. Lett. B276 (1992) 49.

[6] K. S. Stelle, Lectures on Supergravity p-branes, ICTP Summer School, Trieste 1996 [hep-th/9701088].

[7] R. D'Auria, S. Ferrara, M. A. Lledo and V. S. Varadarajan, Spinor Algebras, J. Geom. Phys. 40 (2001) 101 [hep-th/0010124]; R. D'Auria, S. Ferrara and M. A. Lledo, On the Embedding of Space-Time Symmetries into Simple Superalgebras, Lett. Math. Phys. 57 (2001) 123 [hep-th/0102060]; S. Ferrara and M. A. Lledo, Considerations on Superpoincaré Algebras and their Extensions to Simple Superalgebras, Rev. Math. Phys. 14 (2002) 519 [hep-th/0112177].

[8] D. V. Alekseevsky, V. Cortés, C. Devchand and A. van Proeyen, Polyvector Super-Poincaré Algebras, Comm. Math. Phys. bf 253 (2004) 385 [hep-th/0311107].

[9] F. Toppan, Hermitian Versus Holomorphic Complex and Quartenionic Generalized Supersymmetries of the M-Theory: A Classification, JHEP 9 (2004) 016 [hep-th/0406022].

[10] M. F. Atiyah, R. Bott and A. Shapiro, Clifford Modules, Topology (Suppl. 1) 3 (1964) 3.

[11] I. R. Porteous, Clifford Algebras and the Classical Groups, Cambridge Univ. Press, Cambridge 1995.

[12] S. Okubo, Kac-Moody and New Infinite Dimensional Lie Algebras, J. Math. Phys. 32 (1991) 1657; ibid. 32 (1991) 1669.

[13] Z. Kuznetsova and F. Toppan, Constrained Generalized Supersymmetries and Superparticles with Tensorial Central Charges. A Classification, work in preparation.

[14] J. Baez, The Octonions, math.RA/0105155.

[15] H. L. Carrion, M. Rojas and F. Toppan, Quaternionic and Octonionic Spinors: A Classification, JHEP 4 (2003) 040 [hep-th/0302113].

$004 / 13$ 
[16] J. Lukierski and F. Toppan, Generalized Spacetime Supersymmetries, Division Algebras and Octonionic M-Theory, Phys. Lett. B539 (2002) 266 [hep-th/0203149].

[17] J. Lukierski and F. Toppan, Octonionic M Theory and D=11 Generalized Conformal and Superconformal Algebras, Phys. Lett. B567 (2003) 125 [hep-th/0212201].

[18] T. Kugo and P. Townsend, Supersymmetry and the Division Algebras, Nucl. Phys. B221 (1983) 357.

[19] J. Lukierski and F. Toppan, The Symmetry Algebras of Euclidean M-Theory, Phys. Lett. $\mathbf{B 5 8 4}$ (2004) 315 [hep-th/0312083].

[20] E. Sezgin and I. Rudychev, Superparticles, p-Form Coordinates and the BPS Condition, Phys. Lett. B424 (1998) 60 [hep-th/9711128].

[21] I. Bandos and J. Lukierski, Tensorial Central Charges and New Superparticle Models with Fundamental Spinor Coordinates, Mod. Phys. Lett. A14 (1999) 1257 [hep-th/9811022].

[22] I. Bandos, J. Lukierski, C. Preitschopf and D. Sorokin, OSP Supergroup Manifolds, Superparticles and Supertwistors, Phys. Rev. D61 (2000) 065009 [hep-th/9907113].

[23] C. Fronsdal, Massless Particles, Ortosymplectic Symmetry and Another Type of Kaluza-Klein Theory, in "Essays on Supersymmetry", Reidel 1986.

[24] D. Sorokin, Introduction to the Classical Theory of Higher Spin, hep-th/0405069.

[25] M. Hassaine, R. Troncoso and J. Zanelli, Poincaré Invariant Gravity with Local Supersymmetry as a Gauge Theory for the M Algebra, Phys. Lett. B596 (2004) 132 [hep-th/0306258]. 